\title{
Explore the Retail Chain Enterprises Tallyman Turnover Phenomenon
}

\author{
Guangshu Xu, Jie Zhang \& Chao Wang \\ Beijing Wuzi University, Beijing 101149, China
}

\begin{abstract}
Through the questionnaire to survey and analysis the tallyman turnover phenomenon of the retail enterprises in specific areas, make sure the main influence factors that affect the tallyman turnover. After that, applying network maximum flow model making quantitative analysis of the tally clerk turnover management strategy, make a conclude that :relative wages and welfare treatment is an important factor for employee retention; the leadership's attention, encourage and working environmental can motivate staff promotion; relative wages, welfare, leadership's attention and encouragement and working environment can stimulate employees to work; in the promotion and retention management, enterprises should pay more attention to the tally clerk retention management. Finally establish the system cycle diagram to make qualitative analysis and summary.
\end{abstract}

KEYWORD: tallyman turnover; network maximum flow; system cycle diagram

\section{INSTRUCTIONS}

Between the store and virtual shop competition increasing along with the rapid popularization of ecommerce and the rapid development of logistics industry, and nowadays, all kinds of commodities in traditional retail can be purchased through ecommerce platform and been home delivery, which the convenience of combining e-commerce and logistics causing many traditional retail faces enormous challenges. The competitiveness of enterprises, in the final analysis is the talent competition. If companies can implement incentive mechanism effectively, induce the employees work motivation properly, enable them to achieve organizational goals while achieving their own needs, which can promote the rapid development of the enterprise and maintain core competencies to a great extent. As proposed by Wengxian Min [1]: qualities and skills of tallyman are key factors that the supermarket in an invincible position in the fierce market competition. However, there is often a "help wanted" phenomenon because of the front-line staff turnover is high in the retail sector. At present, the related research about tallyman turnover phenomenon is less in our country. For instance, Lihong Guo and Wengxian Min [1-2] just discussed the job responsibilities and management phenomenon of level employees in retail supermarket. Tian Hui and Xia Yu [3-4] from the employer's perspective that employee turnover is a reciprocal behavior and enterprise should take appropriate measures to resist risks what too high staff turnover brings to the enterprise. Li Qing [5] explored and analyzed their turnover reasons and existing solutions mainly for employees of small and medium private enterprises. Wanxiang Lu, Huangyong Tao and others [6-7] proposed incentives for employees' emotions and feelings can increase employee loyalty and enthusiasm. Huanghuo Jun and Qin Song [8] analyzed the underlying problems that lead to retail employee turnover through the actual investigation. But the turnover problems of tallymen have failed to deeply analysis and didn't pay more attention to the occupational demand for level employees in the above studies.

In order to explore the reasons behind that the impact of turnover phenomenon of supermarket tallymen, the large retail supermarket tallymen as the research object in Wuhan. 200 questionnaires were distributed, 152 valid questionnaires recovered, which respectively from basic information of tallymen, tallymen job status, view of the status quo and professional loyalty to research. Through the questionnaire analysis, the turnover problems of tallymen are summarized in the following four aspects: one, the older staff turnover rate is lower; second, the tallymen turnover rate is higher that non- 
resident; third, the attitude of middle managers affect employee turnover rate; fourth, a certain number of employees expect promotion. Taking the needs of level staff as the starting point in this research, analyzes its turnover phenomenon and management of internal promotion (longitudinal turnover) or mobilizing and resigning among in various departments (horizontal turnover). At last, puts forward related suggestions.

\section{TALLYMAN TURNOVER PHENOMENON ANALYSIS}

Employee turnover includes two aspects: the one is interior adjustment, optimal allocation of staff positions and staff mobility; another is employees outflow and inflow of external employees. In this study, the former is called employees transverse flow that is staff from the A zone to B zone and didn't promotion or resignation; the latter is called longitudinal flow that is promotion, a staff can usually from the tallyman promotion to the regional director, then promotion to director assistant, finally to the entire store supervisor. It will not be easily dismissed employees when employer to the employees is unhappy because of the level employees mobility is higher in the retail and the number of recruitment is often inadequate so that retail business recruitment requirements for level staff increasingly reduced, unless their voluntary resignation. In addition, if the supervisor or middle managers whose job performance is not up, coupled with the employers are not satisfied with their work, middle managers generally choose to resign, no demotion. But if an employee wants to promote, their attitude to work and the employer's satisfaction must meet at the same time. In conclusion, the tallymen turnover status can be simplified as Figure1.

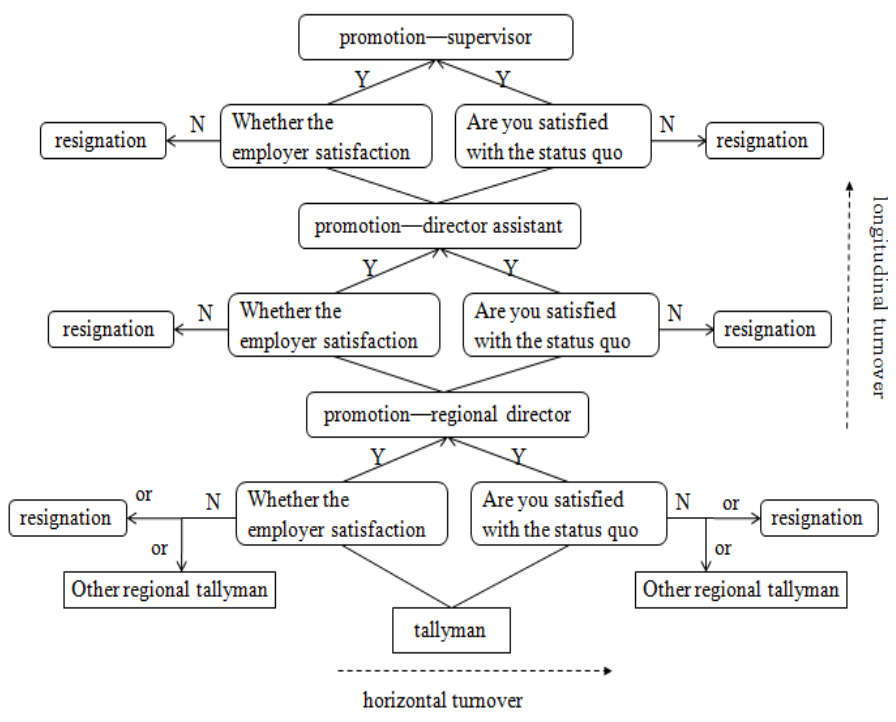

Figure1. Tallyman turnover simplified graph.

\section{TALLMAN TURNOVER MANAGEMENT ANALYSIS}

\subsection{Problem description}

There are capacity constraints in the actual network for nodes and edges, in many cases, if we want to determine the transfer strategies to reach the maximum flow we need to know the flow can be transferred between in a two specified nodes that the node has capacity constraints and respectively called source and sink. In this paper, with the aid of the principle of network maximum flow to establish mathematical model, take the tallymen turnover management measures $v_{1}$ as source, the enthusiasm of tallymen $v_{10}$ as sink. Network nodes include tallyman retention management measures $v_{2}$, tallyman promotion management measures $v_{3}$, relative wage $v_{4}$, welfare benefit $v_{5}$, leadership attention and encouragement $v_{6}$, vocational training $v_{7}$, work environment $v_{8}$ and promotion opportunity $v_{9}$. The capacity constraint between in $i$-th node and $j$-th node is $C_{i j}$, that based on the survey data, the $j$-th node flow is $u_{j}$, and $\theta$ denotes adjustment value. Therefore, the following issues need to be considered in the entire network management system: from tallymen turnover management $v_{1}$ to the enthusiasm of tallymen $v_{10}$ how much management efforts can be put, so that transfer strategy between the various levels can be determined in a capacity constraint management network, that is, each level should be put into management efforts $f_{i j}$ (it's the maximum flow from $i$-th node to $j$-th node ). Network analysis model diagram is established as shown in Figure 2.

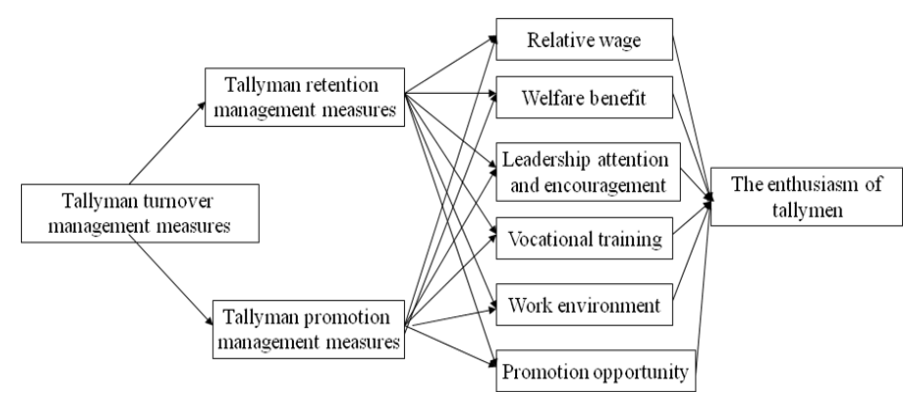

Figure2. Network analysis model diagram

\subsection{The basic steps of Ford-Fulkerson labeling algorithm}

Firstly, found a feasible flow in the network, and given an initial flow valve $f_{i j}=0$, then, found an augmenting path by labeling method, labeled 
$(+,+\infty)$ to $v_{1}$. Secondly, choose a node has label $v_{i}$, using the following method to deal with these nodes which adjacent to $v_{i}$ and have not label: if $f_{j i}>0$, set $u_{j}=\min \left\{f_{j i}, u_{i}\right\}$, and labeled $\left(v_{i}-, u_{j}\right)$ to $v_{j}$; if $f_{i j}<C_{i j}, \quad$ set $u_{j}=\min \left\{C_{i j}-f_{i j}, u_{i}\right\} \quad$, and labeled $\left(v_{i}+, u_{j}\right)$ to $v_{j}$. Repeat the labeling process of previous step until the node $v_{i}$ is labeled or no nodes can be labeled, if $v_{i}$ have been labeled, a feasible augmenting path is going on, and go to the next step, otherwise, there is no such augmenting path, then stop, the current flow $f_{i j}$ is the maximum flow. Thirdly, adjust the flow according to the formula (1) to give a new feasible flow and repeat the three step of above to find new augmenting path until all of nodes will not be labeled.

$$
\begin{gathered}
f_{1}(i, j)=f(i, j) \quad(i, j) \text { is not in augmenting path } \\
f_{1}(i, j)=f(i, j)+\theta \quad(i, j) \text { is forward arc in }
\end{gathered}
$$
augmenting path

$$
f_{1}(i, j)=f(i, j)-\theta \quad(i, j) \text { is backward arc in }
$$
augmenting path

\subsection{Algorithm implementation and result description}

Six factors are taken as the basis of the survey which respectively is relative wages, welfare benefit, leadership attention and encouragements, vocational training, work environment and promotion opportunity, from the perspective of tallyman to analysis the six factors impact on retention, promotion, and working enthusiasm. By statistics and summary the questionnaire data can be drawn from the following data, as shown in Table1.

Table1. The relevant factors statistical table in questionnaire

\begin{tabular}{|c|c|c|c|}
\hline & retention & promotion & enthusiasm \\
\hline relative wages & $53.9 \%$ & $4.6 \%$ & $52.0 \%$ \\
\hline $\begin{array}{c}\text { welfare benefit } \\
\text { leadership attention } \\
\text { and encouragements }\end{array}$ & $20.4 \%$ & $19.7 \%$ & $13.8 \%$ \\
\hline vocational training & $13.8 \%$ & $8.6 \%$ & $19.1 \%$ \\
\hline work environment & $5.3 \%$ & $17.1 \%$ & $9.2 \%$ \\
\hline $\begin{array}{c}\text { promotion } \\
\text { opportunity }\end{array}$ & $4.6 \%$ & $17.8 \%$ & $3.3 \%$ \\
\hline
\end{tabular}

The above data is multiplied by 100 and integer processing, converted into the arc capacity, the matrix of the arc capacity as follows.

$$
\begin{aligned}
& v_{1}\left[\begin{array}{llllllllll}
v_{1} & v_{2} & v_{3} & v_{4} & v_{5} & v_{6} & v_{7} & v_{8} & v_{9} & v_{10} \\
0 & C_{12} & C_{13} & 0 & 0 & 0 & 0 & 0 & 0 & 0
\end{array}\right] \\
& \begin{array}{lllllllllll}
v_{2} & 0 & 0 & 0 & 54 & 20 & 2 & 14 & 5 & 5 & 0
\end{array} \\
& \begin{array}{lllllllllll}
v_{3} & 0 & 0 & 0 & 4 & 20 & 9 & 32 & 17 & 18 & 0
\end{array}
\end{aligned}
$$

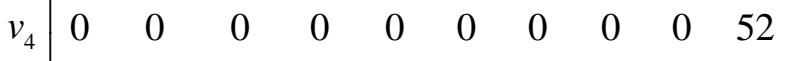

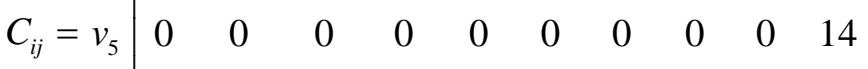

$$
\begin{aligned}
& \begin{array}{l|llllllllll}
v_{6} & 0 & 0 & 0 & 0 & 0 & 0 & 0 & 0 & 0 & 19
\end{array} \\
& \begin{array}{l|llllllllll}
v_{7} & 0 & 0 & 0 & 0 & 0 & 0 & 0 & 0 & 0 & 3
\end{array}
\end{aligned}
$$

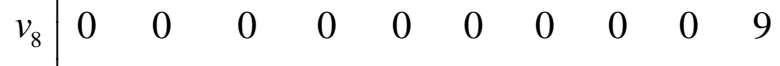

$$
\begin{aligned}
& v_{9} \quad \begin{array}{llllllllll}
0 & 0 & 0 & 0 & 0 & 0 & 0 & 0 & 0 & 3
\end{array} \\
& v_{10}\left[\begin{array}{llllllllll}
0 & 0 & 0 & 0 & 0 & 0 & 0 & 0 & 0 & 0
\end{array}\right]
\end{aligned}
$$

According to the arc capacity matrix, combined with the basic step of Ford-Fulkerson to calculate the maximum flow matrix using Matlab 6.5, the maximum flow matrix is summarized as follows.

$$
\begin{aligned}
& v_{1}\left[\begin{array}{llllllllll}
v_{1} & v_{2} & v_{3} & v_{4} & v_{5} & v_{6} & v_{7} & v_{8} & v_{9} & v_{10} \\
0 & 79 & 13 & 0 & 0 & 0 & 0 & 0 & 0 & 0 \\
0 & 0 & 0 & 52 & 14 & 2 & 3 & 5 & 3 & 0
\end{array}\right] \\
& \begin{array}{l|llllllllll}
v_{2} & 0 & 0 & 0 & 52 & 14 & 2 & 3 & 5 & 3 & 0
\end{array} \\
& \begin{array}{l|llllllllll}
v_{3} & 0 & 0 & 0 & 0 & 0 & 9 & 0 & 4 & 0 & 0
\end{array} \\
& v_{4} \quad \begin{array}{llllllllll}
0 & 0 & 0 & 0 & 0 & 0 & 0 & 0 & 0 & 52
\end{array}
\end{aligned}
$$

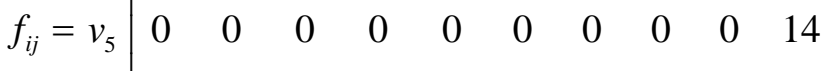

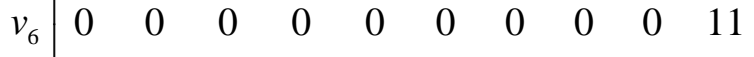

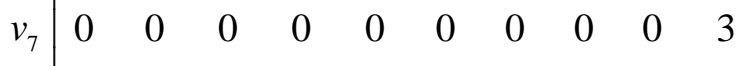

$$
\begin{aligned}
& \begin{array}{l|llllllllll}
v_{8} & 0 & 0 & 0 & 0 & 0 & 0 & 0 & 0 & 0 & 9
\end{array} \\
& v_{9}\left[\begin{array}{lllllllllll}
0 & 0 & 0 & 0 & 0 & 0 & 0 & 0 & 0 & 3
\end{array}\right. \\
& v_{10}\left[\begin{array}{llllllllll}
0 & 0 & 0 & 0 & 0 & 0 & 0 & 0 & 0 & 0
\end{array}\right]
\end{aligned}
$$

In the process of simulation, the maximum flow of the entire system to maintain stability and the flow between the various levels have remained stable when $C_{12}=C_{13}>70$; whether $C_{12}>C_{13}$ or $C_{12}<C_{13}$, the maximum flow of the whole system and the flow between the various levels are also remained stable when $C_{12} 、 C_{13}$, are in the range of changes is greater than 70 . So, if the entire tallyman flow management system to stabilize, the management efforts for tallyman retention is greater than management efforts for tallyman promotion. For tallyman retention, relative wages and benefits are extremely important; the leader's attention and encouragement and working environment plays a decisive role when employees want a promotion; relative wages, benefits, attention and encourage of leadership and working environment are quit important stimulus for stimulating the work enthusiasm of tallyman. All above conclusions derived from Figure 3. 


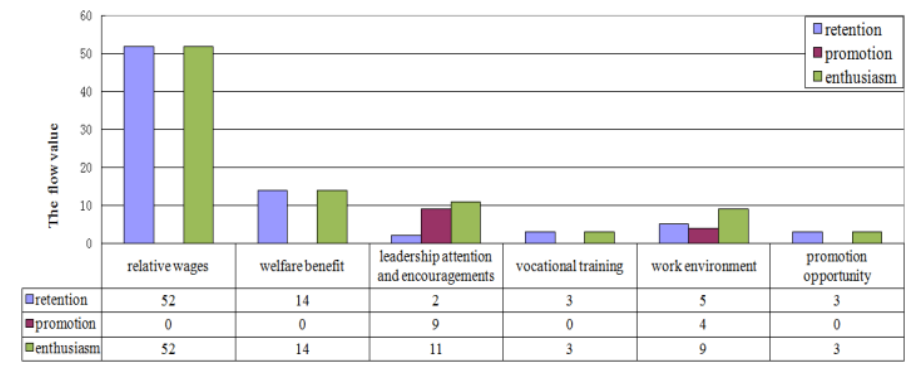

Figure3. simulation results of using Matlab 6.5

\section{ANALYSIS AND SUMMARY}

According to the above analysis combined with the actual research status to draw the related system cycle diagram of tallyman turnover (as shown in Figure 4), there are two circulating loop, one of which is enhanced loop R1 when the motivation is active, the other is control loop B1 when the motivation is negative. So, some conclusions can be summed up:

(1) Enterprises should pay more attention to retention management of tallyman and efforts to implement it by corresponding measures;

(2) Relative wages and welfare benefit play an important role in retention, the enterprises should adjust their tallyman wages and welfare levels appropriate and timely based on the wage movements and benefits updates in the entire retail in order to guarantee the most basic living and welfare of tallyman, which can avoid the negative effects continuing in the B1.

(3) Enterprises should give some attentions to tallyman promotion, appropriate employee turnover can bring "fresh blood" for the enterprise to some extent and make the working environment not immutable and frozen so as to stimulate the work enthusiasm of tallyman that it can make the supermarket is invincible in the fierce market competition.

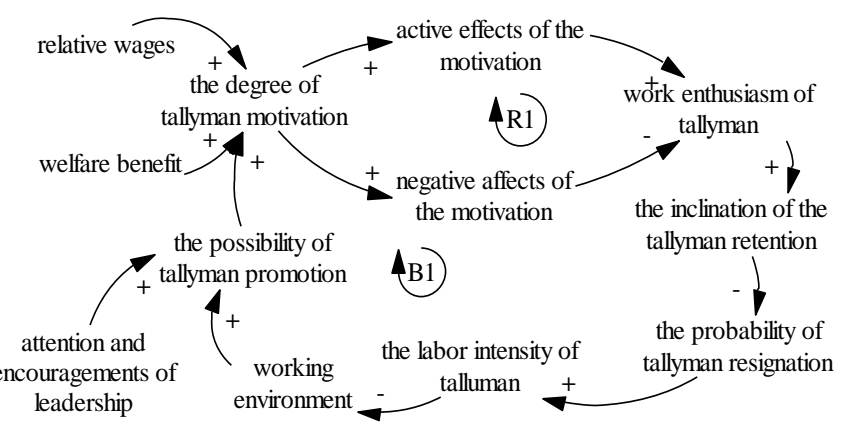

Figure4. System cycle diagram about tallyman turnover

\section{REFERENCES}

[1] Wengxian Ming. Introduction to the supermarket tallyman. Market Modernization, 2005, (25):283-284.

[2] Lihong Guo. From the workflow of tallyman in a day to introduce the employee management. Modern Marketing, 2007, (06).

[3] Wanxiang Lu \& Gujian Ping. Management of retail employees' emotional labor. China Business \& Trade Magazine, 2013, (21):80-81.

[4] Huangyong Tao. Work motivation analysis of supermarket tallyman. Market Modernization, 2006, (10).

[5] Tian Hui. Research in bargaining model of enterprise employee turnover. Enterprise economy, 2012, (02):110113.

[6] Xia Yu. Employee turnover causes analysis and risk control. Business, 2013, (07):48.

[7] Li Qing. Analysis of the reasons and countermeasures of employee turnover in small and medium private enterprise. Guide of Sci-tech Magazine, 2013, (21).

[8] Huanghuo Jun \& Qin Song. Large retail enterprise employee turnover problem research in Wuhan. The Science Education Article Collects, 2006, (07): 197.

[9] Zhangxian Chao et al. Research on the Maximum Network Flow Problem. Journal of Computer Research and Development, 2003, (09): 1281-1289. 